\title{
Combined Single-Step Procedure for Correction of Silent Sinus Syndrome
}

\author{
Roee Arnon ${ }^{a} \quad$ Ofer Gluck $^{b} \quad$ Halit Winter ${ }^{a} \quad$ Joseph Pikkel ${ }^{a} \quad$ Avi Rubinov $^{a}$ \\ aDepartment of Ophthalmology, Assuta-Samson Medical Center, Ben-Gurion University, \\ Ashdod, Israel; 'bepartment of Otolaryngology, Assuta-Samson Medical Center, Ben- \\ Gurion University, Ashdod, Israel
}

\section{Keywords}

Silent sinus syndrome $\cdot$ Single-step procedure

\begin{abstract}
Silent sinus syndrome (SSS) is a rare condition characterized by enophthalmos and hypoglobus that is generally not related to trauma or surgery. We present a 30-year-old man who noticed facial asymmetry during the previous month and was referred to our oculoplastic clinic. His examination revealed right hypoglobus and a 2-mm right enophthalmos. The diagnosis of right SSS was confirmed by orbital and paranasal computed tomography scan. The patient had no otolaryngological symptoms and visual acuity was normal bilaterally. He was treated surgically in a combined approach by a team of oculoplastic and otorhinolaryngology surgeons. Functional endoscopic sinus surgery included uncinectomy, maxillary antrostomy, and orbitotomy, with insertion of an orbital implant. This case demonstrates that a single-step surgery for correction of enophthalmos secondary to SSS is a viable option, leading to quick rehabilitation and excellent aesthetic results.

(C) 2019 The Author(s) Published by S. Karger AG, Basel
\end{abstract}

\section{Introduction}

Silent sinus syndrome (SSS) is a rare clinical condition, characterized by enophthalmos and hypoglobus, which is usually unilateral. The first clinical case was described by Montgomery [1] in 1964. In 1994, Soparkar et al. [2] coined the name of the syndrome and delineated 
it as an idiopathic condition not due to trauma or surgery. More recent reports have referred to the syndrome in describing patients with a history of maxillofacial surgery or trauma $[3,4]$. Other clinical findings include ipsilateral eyelid retraction and deepening of the superior orbital sulcus. Some patients complain of diplopia. Visual acuity and ocular motility are usually preserved [5].

\section{Case Report}

A 30-year-old male presented in the oculoplastic clinic with a complaint of facial asymmetry that had been evident for approximately 1 month. He had no nasopharyngeal symptoms, no allergies or respiratory complaints, and no history of facial trauma or surgeries. He is a smoker and otherwise healthy.

Visual acuity was normal bilaterally; orbital examination revealed mild right hypoglobus and a 2-mm right enophthalmos. Ocular movements were within normal limits without diplopia. The rest of his ophthalmic examination was unremarkable (Fig. 1). SSS was diagnosed by an orbital and paranasal computed tomography scan, which demonstrated volume loss and complete opacification of the maxillary sinus, with bowing and thinning of the right orbital floor (Fig. 2).

The patient underwent a combined surgery by a team of oculoplastic and otorhinolaryngology surgeons. First, using a navigation system, a nasal endoscopic approach was utilized to access the maxillary sinus. An uncinectomy and antrostomy were performed, which revealed a sagging maxillary sinus ceiling, and the sinus was irrigated. A bacterial culture was taken from the extracted mucous. Later, a canthal-sparing transconjunctival approach was utilized to access the orbital floor in the subperiosteal plane. In order to prevent the spread of any potential infection from the sinus, the orbital floor was not opened. A Su-Por ${ }^{\circledR}$ regular right enophthalmos wedge surgical implant $(24 \times 33.5 \times 7 \mathrm{~mm})$ was used to correct the hypoglobus and enophthalmos. In the first few days after surgery, the patient reported mild diplopia in the frontal gaze, which resolved spontaneously. A maxillary mucous culture was positive for Citrobacter freundii and Morganella morganii; both were found sensitive to a short course of oral ciprofloxacin. The patient was discharged 1 day after the surgery. At examination 8 weeks postoperatively, the enophthalmos had resolved completely, and there was no diplopia or limitation of gaze (Fig. 3).

\section{Discussion}

The SSS equally affects both genders, usually between the 3rd and 5th decades of life [4, 5]. Our patient had no ophthalmic history, no recent trauma or surgery, and no medical illness.

There are two leading theories for the pathogenesis of SSS. The "obstruction of outflow" theory posits that acquired obstruction of the maxillary infundibulum causes hypoventilation and accumulation of secretions. Once the secretions have reabsorbed, negative pressure in the sinus leads to inward retraction of the sinus walls, including the orbital floor, and bone resorption due to increased osteoclastic activity. Due to thinning and weakening of the orbital floor, the paucity of orbital support results in hypoglobus and enophthalmos [2, 4-8]. According to the second theory, known as the "mechanical" theory, the contraction and relaxation of masticatory muscles may cause aspiration of a closed maxillary antrum, leading to negative pressure within the sinus and collapse of its walls [4]. 
Our patient's complaint was mainly facial asymmetry, without any nasopharyngeal symptoms or pain. This concurs with other reports that showed that patients with SSS usually present in the ophthalmological clinic and complain of facial asymmetry, with little or no nasopharyngeal symptoms $[6,9]$. The ophthalmic examination typically reveals unilateral, painless, hypoglobus (range 2-6 $\mathrm{mm}$ ) and enophthalmos (range 2-5 $\mathrm{mm}$ ), upper eyelid retraction, and deepening of the superior orbital sulcus [2]. As with our patient, visual acuity and ocular motility are usually preserved. Most patients do not complain of diplopia [5]. The name "silent" is derived from the painless course and the slow development [6]. Though rare, SSS can be bilateral [10] or accompanied with symptoms such as headache, facial pain, and post-nasal drip [11].

SSS is diagnosed clinically, though a high index of suspicion is required in some cases. SSS is confirmed with orbital and paranasal computed tomography scans, which are considered the gold standard [6]. Typical findings of SSS that appear in imaging include (1) maxillary sinus obstruction, usually by lateral retraction of the uncinate process, with apposition against the inferomedial aspect of the orbital wall and (2) enlargement of the middle meatus, with abnormal opacification of the maxillary sinus, accompanied by inward retraction and volume decrease. The orbital floor is retracted inferiorly to the sinus lumen in a "bow" shape, which results in increased orbital volume and inferior displacement of the globe and orbital content $[5,11-13]$.

Treatment of SSS is aimed at two main goals, sinusoidal and orbital. The sinusoidal goal is dilatation of the maxillary ostium to achieve sinus aeration and restore normal drainage. The orbital goal is reconstruction of the orbital floor to correct hypoglobus and enophthalmos. First, the maxillary ostium is dilated endoscopically by antrostomy and uncinectomy. Subsequently, using a transconjunctival or subciliary approach, an alloplastic or autogenous implant is inserted to stabilize the orbital floor. As in our patient, an image-guided navigation system can aid in the positioning of the endoscope and reducing the risk of orbital floor injury, which is lower than normal [5].

The timing and opting for orbital floor correction are a matter of debate. According to some reports, correction should be done in two stages. Aeration of the maxillary sinus sometimes suffices to improve enophthalmos and hypoglobus. Therefore, some argue for waiting a few months before addressing the orbit. Other advantages of this two-stage approach are avoidance of the risk of infection of the implant and of overcorrecting the globe position [5, 14]. Other data support a single-step correction, with the rationale of preventing complications of a second general anaesthesia and minimizing hospital stay [8]. We conducted a singlestep procedure of endoscopic surgery and orbital reconstruction to minimize patient discomfort and with the aim of rapid rehabilitation. Even though sinus cultures were positive, there was no evidence of implant infection postoperatively during the follow-up period of 2 months, and recovery was complete and uneventful. A single-step correction using balloon sinuplasty technique for maxillary sinus aeration was recently reported [15].

\section{Conclusion}

Concurring with other reports, the current patient presented in the ophthalmological clinic with SSS, and without any otolaryngological symptoms. SSS should be included in the differential diagnosis for facial asymmetry. Although the surgical stages for the repair of SSS can be separated, for the patient described, a successful multidisciplinary single-step procedure resulted in quick rehabilitation and excellent aesthetic results. 


\section{Statement of Ethics}

The authors have no ethical conflicts to disclose. Written informed consent was obtained from the patient.

\section{Disclosure Statement}

The authors have no conflicts of interest to declare.

\section{Funding Sources}

This work received no financial support.

\section{Author Contributions}

Dr. Arnon wrote and reviewed the manuscript and assisted during surgery. Dr. Rubinov is the head of oculoplastic surgery and reviewed the manuscript. Dr. Gluck is the head otorhinolaryngology surgeon. Prof. Pikkel reviewed the manuscript. Dr. Winter reviewed the manuscript. All authors read, edited, and approved the case report.

\section{References}

1 Montgomery WW. Mucocele of the maxillary sinus causing enophthalmos. Eye Ear Nose Throat Mon. 1964 May;43:41-4.

2 Soparkar CN, Patrinely JR, Cuaycong MJ, Dailey RA, Kersten RC, Rubin PA, et al. The silent sinus syndrome. A cause of spontaneous enophthalmos. Ophthalmology. 1994 Apr;101(4):772-8.

3 Canzi G, Morganti V, Novelli G, Bozzetti A, Sozzi D. Posttraumatic Delayed Enophthalmos: Analogies with Silent Sinus Syndrome? Case Report and Literature Review. Craniomaxillofac Trauma Reconstr. 2015 Sep;8(3):251-6.

4 Cobb AR, Murthy R, Cousin GC, El-Rasheed A, Toma A, Uddin J, et al. Silent sinus syndrome. Br J Oral Maxillofac Surg. 2012 Sep;50(6):e81-5.

5 Annino DJ Jr, Goguen LA. Silent sinus syndrome. Curr Opin Otolaryngol Head Neck Surg. 2008 Feb;16(1):225.

6 Guillen DE, Pinargote PM, Guarderas JC. The silent sinus syndrome: protean manifestations of a rare upper respiratory disorder revisited. Clin Mol Allergy. 2013 Dec;11(1):5.

7 Gan WL. Silent sinus syndrome as a recognised cause of unilateral painless enophthalmos. BMJ Case Rep. 2014 May;2014(may23 1):2014.

8 Sesenna E, Oretti G, Anghinoni ML, Ferri A. Simultaneous management of the enophthalmos and sinus pathology in silent sinus syndrome: a report of three cases. J Craniomaxillofac Surg. 2010 Sep;38(6):469-72.

9 Vander Meer JB, Harris G, Toohill RJ, Smith TL. The silent sinus syndrome: a case series and literature review. Laryngoscope. 2001 Jun;111(6):975-8.

10 Trope M, Schwartz JS, Tajudeen BA, Kennedy DW. Bilateral silent sinus syndrome: A rare case and review of literature. Allergy Rhinol (Providence). 2017 Jun;8(2):100-2.

11 Ferro A, Basyuni S, Santhanam V. Not so silent sinus syndrome: A case report. Int J Surg Case Rep. 2016;23:1-3 


\section{Case Reports in Ophthalmology}

12 Rose GE, Sandy C, Hallberg L, Moseley I. Clinical and radiologic characteristics of the imploding antrum, or "silent sinus," syndrome. Ophthalmology. 2003 Apr;110(4):811-8.

13 Illner A, Davidson HC, Harnsberger HR, Hoffman J. The silent sinus syndrome: clinical and radiographic findings. AJR Am J Roentgenol. 2002 Feb;178(2):503-6.

14 Thomas RD, Graham SM, Carter KD, Nerad JA. Management of the orbital floor in silent sinus syndrome. Am J Rhinol. 2003 Mar-Apr;17(2):97-100.

15 Kashima T, Goldberg RA, Kohn JC, Rootman DB. One-stage bone strip reconstruction technique with balloon sinus dilatation surgery for chronic maxillary atelectasis. Clin Ophthalmol. 2016 Nov;10:2363-8.
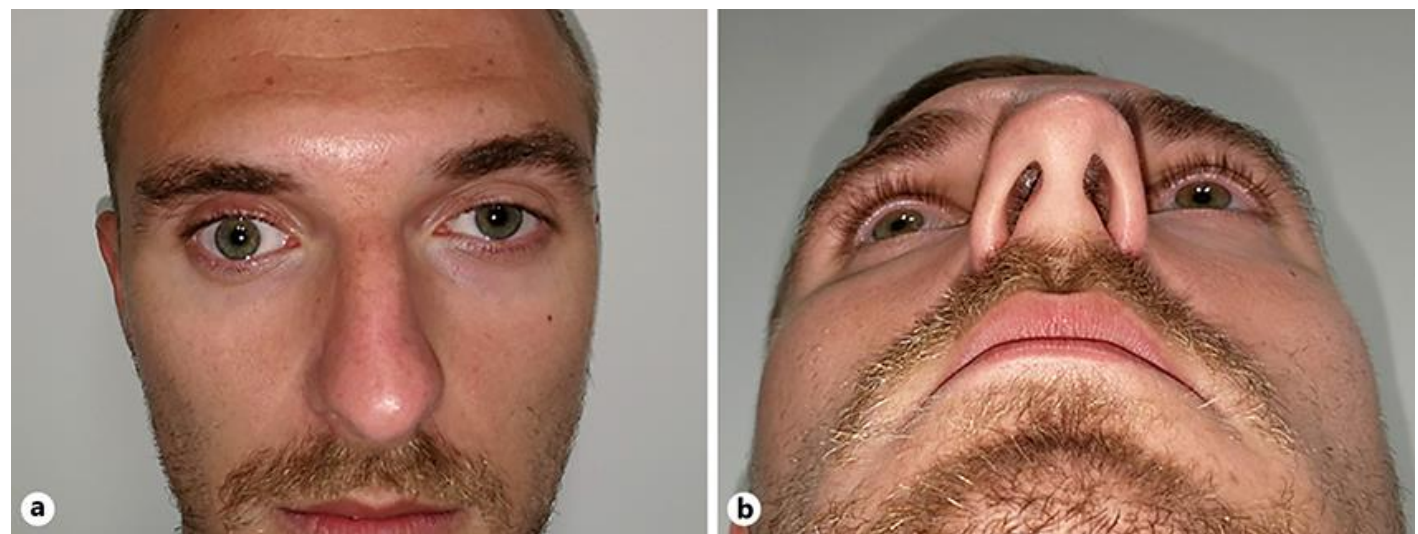

Fig. 1. a Preoperative frontal view of the patient showing right-sided hypoglobus, upper eyelid retraction, and deepening of the superior orbital sulcus. b Preoperative worm's-eye view showing right-sided enophthalmos.

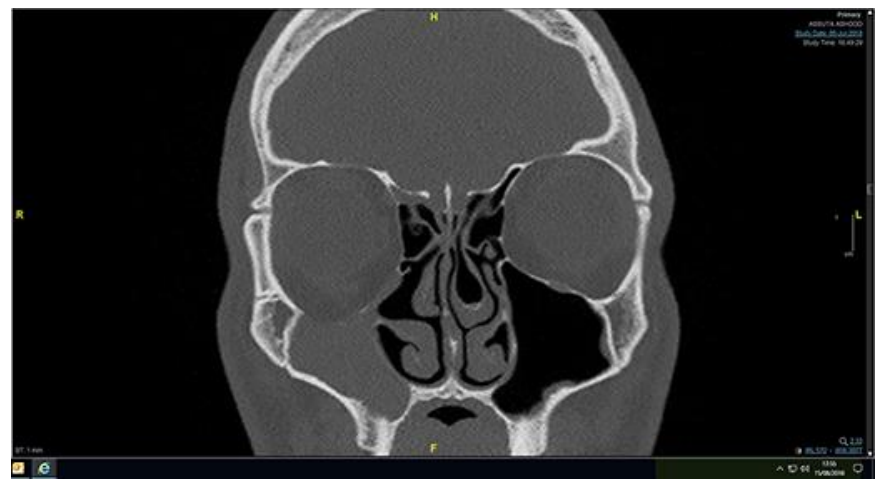

Fig. 2. Coronal CT scan showing orbital floor bowing and maxillary sinus opacification. 


\section{Case Reports in Ophthalmology}
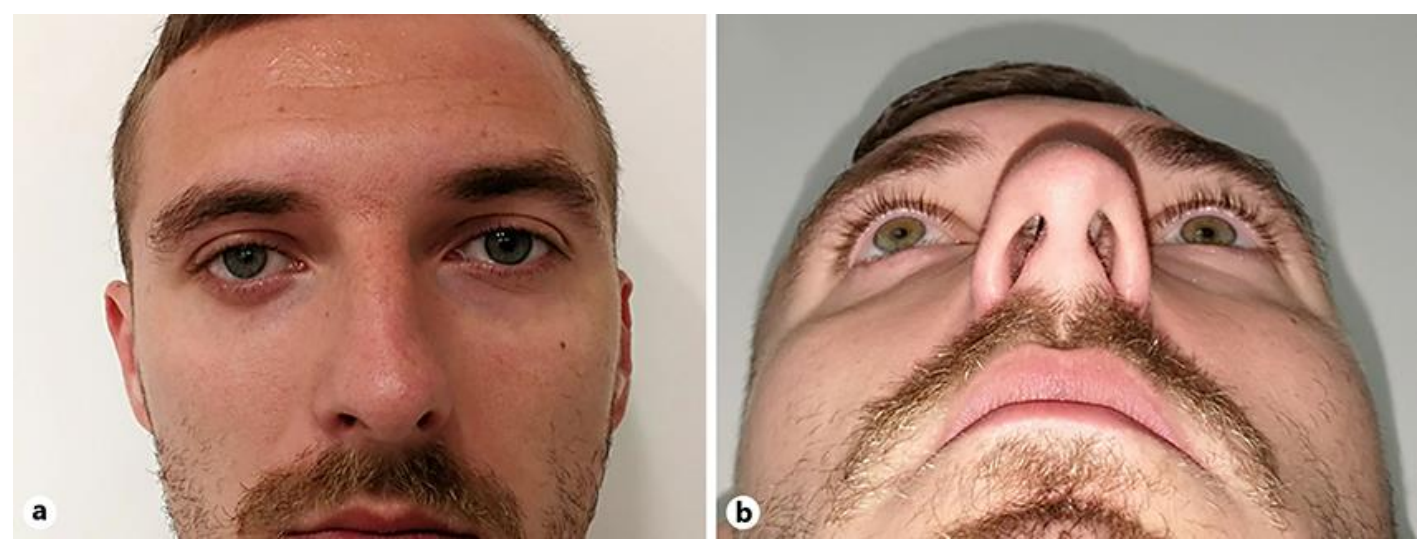

Fig. 3. Eight-week postoperative view. a Frontal view of the patient showing resolution of hypoglobus, upper eyelid retraction, and deepening of the superior orbital sulcus. b Worm's-eye view showing resolution of right-sided enophthalmos. 